\title{
Skin Appendage Neoplasm
}

National Cancer Institute

\section{Source}

National Cancer Institute. Skin Appendage Neoplasm. NCI Thesaurus. Code C4463.

A benign or malignant neoplasm that arises from the hair follicles, sebaceous glands, or sweat glands. 